Mots. Les langages du politique

$86 \mid 2008$

Toponymes. Instruments et enjeux

\title{
La tête de Nicolas Sarkozy, ou les fonctions des gestes de la tête durant l'énonciation
}

Geneviève Calbris

\section{(2) OpenEdition \\ Journals}

Édition électronique

URL : https://journals.openedition.org/mots/14002

DOI : $10.4000 /$ mots. 14002

ISSN : 1960-6001

Éditeur

ENS Éditions

Édition imprimée

Date de publication : 30 mars 2008

Pagination : 99-118

ISBN : 978-2-84788-129-5

ISSN : 0243-6450

Référence électronique

Geneviève Calbris, "La tête de Nicolas Sarkozy, ou les fonctions des gestes de la tête durant l'énonciation », Mots. Les langages du politique [En ligne], 86 | 2008, mis en ligne le 30 mars 2010, consulté le 23 avril 2022. URL : http://journals.openedition.org/mots/14002 ; DOI : https://doi.org/ $10.4000 /$ mots. 14002

\section{(C) ENS Éditions}




\section{La tête de Nicolas Sarkozy, ou les fonctions des gestes de la tête durant l’énonciation}

Cet article a pour but de montrer que les fonctions communicatives et énonciatives généralement effectuées au sein de la parole par l'ensemble des mouvements du corps peuvent l'être par les seuls mouvements de tête. Autrement dit, la communication non verbale visuelle passe déjà en gros plan. Il s'agira moins de la fonction expressive des mouvements des muscles du visage que de la fonction référentielle des mouvements de la tête, analogue à celle des mouvements des bras. L'article ne traite pas de la communication politique à l'écran et n'étudie pas non plus en particulier celle du locuteur.

Pour illustrer cette étude, nous nous appuierons sur une partie de l'interview de Nicolas Sarkozy sur la chaîne régionale FR3, au journal télévisé de 19h-20h, le 26 mai 2005.

\section{Remarques préliminaires}

\section{Gestes de la tête}

En dehors des signes occidentaux de négation et d'affirmation susceptibles de remplacer la parole, les gestes de la tête sont relativement ignorés. Certains gestualistes ont étudié les fonctions et les significations de mouvements de tête particuliers, tels Adam Kendon qui a analysé de nombreux emplois du secouement latéral de la tête en Angleterre et en Italie (Kendon, 2002), et Evelyn McClave qui propose une comparaison interculturelle de l'utilisation de la tête dans l'énumération et la deixis (McClave, 2005).

Un classement physique, et même physico-sémantique, des mouvements de tête durant la parole a été entrepris à partir de conversations observées sur le terrain il y a déjà plus de vingt ans en France (Calbris, 1983, p. 694-760). Bien que reliée au cou, la tête se déplace en ligne droite sur les divers axes, par exemple sur l'axe vertical, vers le haut en allongeant le cou pour mieux

CNRS (LEAPLE), calbris@vjf.cnrs.fr, calbris.genevieve@free.fr 
voir ou se faire voir (haussement) ou bien, à l'inverse, vers le bas en rentrant la tête dans les épaules pour mieux se cacher. Par ailleurs, la tête est assimilable à une sphère susceptible d'exécuter différents mouvements en ligne courbe. Ceux-ci correspondent à ses diverses possibilités d'évolution: en plan sagittal, vers le haut (relèvement) ou vers le bas (abaissement); en plan frontal, en penchant la tête vers l'épaule droite ou l'épaule gauche (penchement); en plan horizontal, sur l'axe transversal (rotation). Il va sans dire qu'on peut assister à une combinaison de mouvements: par exemple, à un relèvement de la tête en diagonale sur le côté; ou à une rotation transversale alliée à un penchement comme pour mieux regarder par en dessous l'objet qui fait face. Ce sont effectivement les divers mouvements rencontrés dans le corpus annoté ci-dessous.

L'identification des mouvements de tête est parfois difficile et leurinterprétation est également malaisée. Sur l'ensemble des répertoires de signes gestuels coverbaux français progressivement constitués, il a fallu s'appuyer sur les phénomènes de redondance ou de substitution constatés entre les gestes de la main et ceux de la tête pour élucider ces derniers, passant des premiers, lisibles, aux seconds, moins lisibles mais mis en équivalence. Par exemple, l'exclamation augmentative, déjà vocalement signifiée par une montée mélodique, est signifiée par un mouvement vers le haut de la tête et / ou du sourcil et / ou de la main levée de profil. De même, le mouvement transversal figurant la ligne d'horizon en référence à la totalité ('tout', 'partout') ${ }^{1}$ peut être effectué avec la main recouvrant tout l'horizon et / ou avec la tête le balayant du regard. C'est ainsi que de nombreuses informations non verbales des gestes de la main, obtenues en plan américain, se retrouvent dans ceux de la tête du locuteur pris en gros plan.

\section{Quelques clés pour l'analyse gestuelle dans la communication orale}

La communication orale est multicanale: elle passe par le canal verbal et par deux autres canaux dits non verbaux, le canal vocal (la prosodie) et le canal kinésique, lui-même multiple (la posturo-mimo-gestualité2). Non seulement la communication orale passe par plusieurs canaux, mais chaque canal assure plusieurs fonctions.

Les fonctions communicatives sont centrées sur le locuteur (fonction expressive), le destinataire (fonction d'appel) et le message (fonction référentielle), mais aussi sur le maintien de la communication (fonction phatique). Communicatif, le geste assure les mêmes fonctions.

1. Par convention scripturale, les paraphrases des signifiés gestuels correspondant à ces divers mouvements sont proposées entre guillemets simples.

2. Terme proposé par Jacques Cosnier pour dénommer les mouvements des différents segments corporels. 
Coverbal, le geste assure aussi les fonctions énonciatives concernant la rythmisation de la parole (fonction démarcative) et sa mise en mots (fonction énonciative) car, en tant qu'esquisse concrète et métaphorique de l'idée, il annonce souvent ce qui va être dit.

Tableau 1. Multifonctionnalité de chaque canal

\begin{tabular}{|c|c|c|c|c|c|c|}
\hline \multirow{2}{*}{ Canal } & \multicolumn{3}{|c|}{ Fonctions communicatives } & \multicolumn{2}{c|}{ Énonciatives } \\
\hline & expressive & d'appel & référentielle & phatique & démarcative & énonciative \\
\cline { 2 - 7 } & & & & & rythmisation & mise en mots \\
\hline verbal & - & - & - & - & & \\
\hline vocal & - & - & & - & - & \\
\hline kinésique & - & - & - & - & - & - \\
\hline
\end{tabular}

Si le canal vocal et le canal kinésique s'associent pour assurer la plupart des fonctions, seul le geste peut, comme en langue des signes, avoir une fonction référentielle. Le geste assure le plus grand nombre de fonctions et c'est en tenant compte à la fois de la multicanalité de la communication et de sa multifonctionnalité éventuelle qu'on étudie sa fonction référentielle pour constater que c'est un signe à la fois contextuel, polysémique et toujours motivé: il présente un lien analogique naturel entre son aspect physique et sa signification contextuelle.

L'interprétation correcte du geste suppose, certes, une prise en compte du contexte situationnel, verbal, vocal, kinésique (autres mouvements corporels), mais aussi une connaissance du système de signes gestuels et de leur fonctionnement symbolique. Ainsi la polysémie d'un geste s'explique-t-elle non pas uniquement par une dérivation sémantique à partir d'une signification physique essentielle (motivation unique), mais aussi et souvent par la présence de plusieurs liens analogiques possibles (motivation plurielle) sur lesquels se greffe une dérivation sémantique (Calbris, 1987).

Le contexte sélectionne une des potentialités physico-symboliques du geste révélées par la comparaison des diverses significations contextuelles observées. Le cas de la tête penchée en est un exemple. Le penchement peut être: soit un mouvement déictique vers un point situé à gauche ou à droite, sur un axe transversal spatio-temporel (mouvement déictique sur la gauche référant à un événement antérieur); soit la figuration d'une diagonale par rapport à un axe vertical; soit une posture d'attendrissement: on penche la tête vers l'épaule comme pour demander à reposer sa tête sur l'épaule d'autrui (Morris, 1987, p. 48; Montagner, 1978, p. 282); soit la reproduction d'un mouvement pour voir sous un autre angle et figurer ainsi un autre point de vue apporté sur la chose, un point de vue particulier, personnel ou restrictif (quatorze exemples dans Calbris, 1983, p. 730-731), etc. 
Bref, la prise en compte de la multicanalité permet non seulement de désambigüiser les éléments en interaction sur les divers canaux, mais aussi de synthétiser les informations de nature sémiotique différente, le geste formulant concrètement et, par là même, annonçant l'idée à mettre en mots, à moins qu'il ne précise, n'explicite ou ne commente l'information sonore simultanément énoncée.

\section{Corpus}

Le 26 mai 2005, donc, sur FR3, Nicolas Sarkozy est interviewé par deux journalistes, Stéphane Lippert et Jean-Michel Blier, à l'occasion du référendum sur la Constitution européenne. Mais tous deux commencent par l'interroger sur les rumeurs concernant sa situation familiale. Le texte ci-dessous ne rapporte que le début de cette interview, dont l'Agence France Presse a prélevé trois moments pour effectuer un montage d'extraits (en italiques) proposés, à l'époque, sur le site internet Voilà. L'analyse de ce montage suffira à la démonstration de l'apport sémantique des mouvements de tête.

\section{L'interview}

Stéphane Lippert interroge Nicolas Sarkozy à propos de sa fatigue et de la rumeur concernant ses problèmes conjugaux).

N. S. : Vous voulez parler de la rumeur, mais interrogez-moi alors, faites votre joli métier, allez jusqu'au bout. Quelle est la réponse que vous voulez que je vous apporte?

S. L. : Je ne sais pas, quelle est la vérité?

N. S. : La vérité sur quoi ? La vérité c'est que je suis un homme public lancé dans une campagne électorale en faveur du Oui. Je fais campagne tous les soirs. Il est normal qu'en tant qu'homme public, je réponde aux questions. Mais est-ce que vous ne trouvez pas que parfois ça va un peu loin et que ma famille mérite aussi d'être respectée, que ma femme, qui est quelqu'un d'éminemment respectable, mérite aussi d'être respectée, qu'elle n'a pas à être suivie en permanence par des motos avec des photographes pour savoir avec qui elle déjeune, que mes enfants doivent être préservés? Alors [la vérité, elle est très simple comme des millions de familles, la mienne a connu des difficultés. Ces difficultés, nous sommes en train de les surmonter. Est-ce que je dois en dire plus? Je ne le pense pas]. Et ma famille, elle a besoin d'un peu de répit et je demande simplement que ma famille soit respectée. Moi je suis un homme politique, je peux combattre, je peux me défendre, eux pas. Alors voyez-vous, je me suis longuement interrogé pour savoir si je devais répondre à cette question. Les rumeurs les plus folles ont couru sur une maladie, une dépression, des situations invraisemblables qui seraient arrivées aux miens. Je demande simplement d'être respecté, voilà. Je ne me plains pas, j'assume, je vous ai dit ce que j'avais à dire, il n'y a rien à dire de plus. 
Stéphane Lippert fait remarquer à Nicolas Sarkozy la mise en avant du rôle de sa femme dans sa carrière.

N. S.: «Bien sûr c'est, vous savez, je ne suis pas le premier à travailler avec son épouse [Cécilia, je lui dois énormément. Cécilia compte beaucoup pour moi, chacun le sait. Je crois que la réciproque est également vraie. Je ne pense pas que cela mérite qu'on dise des choses, la plupart du temps d'ailleurs inexactes, qui lui manquent beaucoup de respect et qui la font souffrir.] C'est tout, je n'ai rien d'autre à dire.

Jean-Michel Blier insiste sur la rapidité de la rumeur.

N. S.: Quelle affaire, Monsieur Blier, y a aucune affaire, y a une famille qui essaie de construire. [Et malheureusement, nous vivons dans un monde - je ne m'en plains pas, je dis les choses telles qu'elles sont - où tout le monde n'a pas les mêmes scrupules, où tous les coups peuvent être donnés et où pour abattre quelqu'un, on utilise tous les procédés. Je reste serein et je reste calme. Je fais cet engagement qui est le mien depuis bien longtemps: rien ne me détournera de la route que j'ai choisie.] Je crois dans mes idées. La seule chose qui m'interroge et qui à mon tour me fait souffrir, c'est quand on touche à ceux que j'aime le plus, parce j'en porte une part de responsabilité puisque je suis le chef de cette petite famille, je dois les protéger et c'est ça la seule chose qui compte. Pour le reste, je n'ai rien d'autre à dire [...].

Les trois interlocuteurs sont répartis autour d'une table en arc de cercle presque fermé. Stéphane Lippert est au centre, Nicolas Sarkozy à gauche sur l'écran et Jean-Michel Blier à droite. Compte tenu de cette disposition, Nicolas Sarkozy penche un peu la tête à droite pour répondre au premier journaliste sur sa gauche et, inversement, à gauche pour répondre au second qui est sur sa droite. Dans les deux premiers extraits, Nicolas Sarkozy l'homme politique s'adresse à Stéphane Lippert sur sa gauche et dans le troisième, à Jean-Michel Blier sur sa droite. Ces positions de tête tributaires du contexte physique n'ont pas de signification.

\section{Codage des extraits vidéo}

\section{Canal vocal}

Le codage signale essentiellement les éléments prosodiques sur lesquels s’appuie l'analyse du canal kinésique.

Codage adopté pour les éléments prosodiques (P.) :

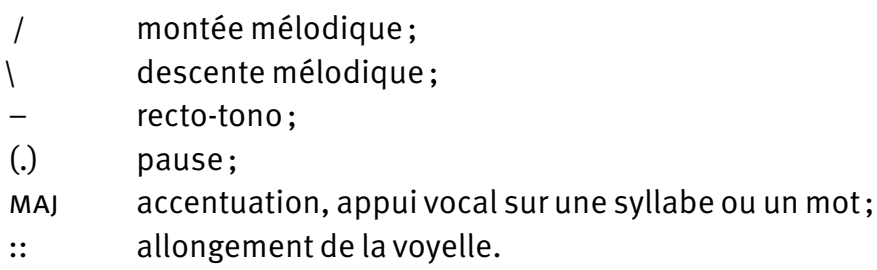


Le corpus analysé ne contient aucune hésitation ni reprise. Seules les informations intonatives particulièrement pertinentes sont annotées, à savoir les mouvements de montée (/), de descente ( $($ ), le palier recto tono (-) correspondant aux incises. La pause (.) en fin de groupes rythmico-sémantiques sépare les unités de sens, mais lorsqu'elle survient au sein d'un groupe, elle permet de mettre la suite attendue en relief (segments 9, 10). Autres mises en relief possibles, la syllabation (segment 6) ainsi que l'accent vocal codé par des majuscules (maj) sur la syllabe accentuée (segments 3, 8, 10, 16, 18, 19, 20). L'accent va de pair avec un pic mélodique (segments $3,8,18$ ) ou est parfois renforcé par un mouvement d'appui de la tête vers le bas: nous avons alors affaire à une double accentuation, voco-kinésique (cf. segments 10,19).

\section{Canal kinésique}

Le découpage sonore prosodique assuré par les mouvements mélodiques différents et les pauses est corroboré par le découpage kinésique. Les moments de changement dans les mouvements cooccurrents du corps - décrits ou codés ci-dessous - mettent en évidence la segmentation kinésique hiérarchisée du discours (Condon, Kendon, 1972), depuis l'expression d'une idée au sein d'une phrase, le groupe rythmico-sémantique, jusqu'à la mise en exergue d'un mot, comme on peut le constater ci-dessous.

La description codée des mouvements de divers segments corporels (tête, regard, sourcils, bouche) figure sous les segments verbaux simultanément énoncés, numérotés pour faciliter ensuite leur repérage.

Codage adopté pour signaler les changements des mouvements corporels (lieu du mouvement: le tronc [Tr], la tête $[T]$; au niveau du visage : la bouche $[B]$, le regard [R], les sourcils [S]) :

i tête et / ou regard orientés vers l'interlocuteur;

$\wedge \wedge \quad$ haussement des sourcils;

$\wedge \quad$ haussement d'un sourcil, du seul sourcil droit sur l'ensemble du corpus;

$>\quad$ sourcils froncés;

(:) sourire fermé;

... maintien de la tête dans la position adoptée;

repos retourà la position initiale de repos. 


\section{Extrait 1 : Nicolas Sarkozy s’adresse à Stéphane Lippert sur sa gauche.}

1. La vérité, elle est très simple. Comme des millions de familles,

$\begin{array}{ccccc}\mathrm{P} & & / & / & \text { (.) } \\ \mathrm{T} & \text { en bas } & \text { relevée, mouv. asc. surla gauche vers } \mathrm{i} & & \text { s'abaisse } \\ \mathrm{R} & \text { en bas } & \mathrm{i} & & \\ \mathrm{S} & & \wedge \wedge & \end{array}$

2.

la mienne a connu des difficultés.

\begin{tabular}{lccc}
$\mathrm{P}$ & $/$ & 1 & $()$. \\
$\mathrm{T}$ & secouée & \\
$\mathrm{R}$ & \multicolumn{1}{l}{} & \\
$\mathrm{B}$ & petit sourire &
\end{tabular}

3. Ces difficultés, nous sommes EN TRAIN de les surmonter.

\begin{tabular}{|c|c|c|c|c|c|}
\hline$P$ & & & I & 1 & (.) \\
\hline $\operatorname{Tr}$ & rehaussé & &. & repos & \\
\hline $\mathrm{T}$ & rehaussée & &. & repos & \\
\hline $\mathrm{R}$ & fermé & & & & \\
\hline$S$ & $\wedge \wedge$ & & 1 & & \\
\hline & Est-ce que je dois & en dire & plus? & & \\
\hline$P$ & I & 1 & I & (.) & \\
\hline $\mathrm{T}$ & relevée vers i & repos & relevée vers i & recentrée & \\
\hline$R$ & fermé & i & $\mathrm{i}$ & & \\
\hline S & $\wedge \wedge$ & & $><$ & & \\
\hline & Je nele pense & & pas. & & \\
\hline$P$ & I & & (.) & & \\
\hline $\mathrm{T}$ & $\begin{array}{l}\text { détournée à gauche } \\
\text { et très penchée à droite }\end{array}$ & & $\begin{array}{l}\text { recentrée } \\
\text { abaissée }\end{array}$ & & \\
\hline $\mathrm{R}$ & $\mathrm{i}$ & & i & & \\
\hline
\end{tabular}

\section{Extrait 2}

6. Cé-ci-lia, je lui dois énormément.

$\begin{array}{lcc}\mathrm{P} & \text { centrée, immobile } & (.) \\ \mathrm{T} & \text { fixe devant soi } & \\ \mathrm{R} & & \\ \mathrm{B} & & \text { commissures étirées }\end{array}$




\begin{tabular}{cccccc}
\hline 7. & Cécilia compte beaucoup & pour moi, & chacun & le sait. & \\
\hline $\mathrm{P}$ & & $/$ & - & - & $()$. \\
$\mathrm{T}$ & baissée à gauche & relevée vers $\mathrm{i}$ & & recentrée & \\
$\mathrm{R}$ & bas & $\mathrm{i}$ & $\mathrm{i}$ & vers le bas & \\
$\mathrm{B}$ & sourire & sourire audible & sourire & & \\
& & & & \\
\hline
\end{tabular}

\begin{tabular}{ccc}
\hline 8. & Je crois que la réciproque est également VRAIE. \\
\hline $\mathrm{P}$ & / (.) \\
$\mathrm{T}$ & centrée, immobile \\
$\mathrm{R}$ & fixe devant soi \\
\hline
\end{tabular}

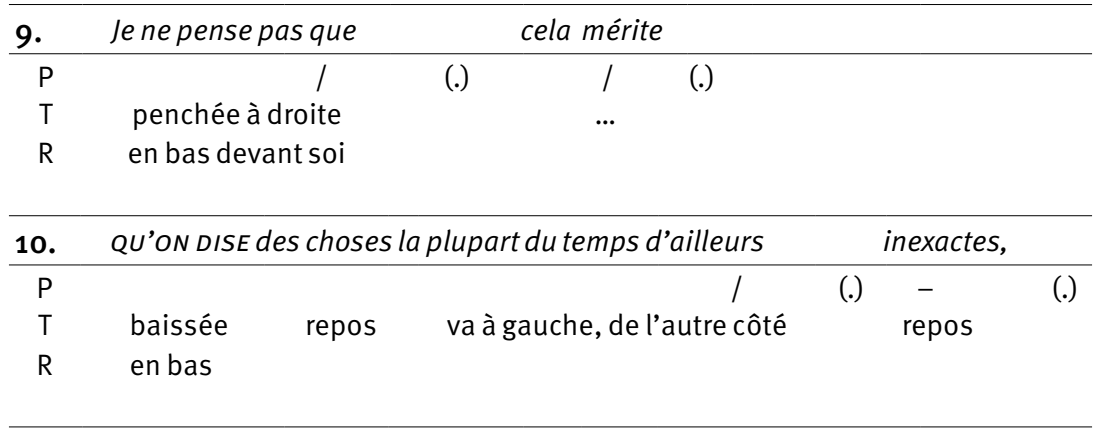

\begin{tabular}{rcc}
\hline 11. & quilui manquent beaucoup de respect & et qui la font souffrir. \\
\hline $\mathrm{P}$ & deux fois abaissée & ton traînant::: \\
$\mathrm{T}$ & en bas & tournée vers $\mathrm{i}$ \\
$\mathrm{R}$ & & $\mathrm{i}$ \\
$\mathrm{B}$ & & commissures étirées
\end{tabular}

\begin{tabular}{cccc}
\hline & C'est tout, & je n'ai rien d'autre à dire. \\
\hline $\mathrm{P}$ & (faible intensité) & (.) & (et rythme rapide exprimant la lassitude) (.) \\
$\mathrm{T}$ & aller-retour transv. & secouée \\
$\mathrm{R}$ & $\mathrm{i}$ & $\mathrm{i}$
\end{tabular}

\section{Extrait 3 : Nicolas Sarkozy s’adresse à Jean-Michel Blier sur sa droite.}

12. Et malheureusement, euh...nous vivons dans un monde -je ne m'en plains pa-as,

\begin{tabular}{|c|c|c|c|}
\hline $\mathrm{P} \quad /:$ & l & & ton au-dessus, plaintif (.) \\
\hline $\mathrm{T}$ & centrée, baissée & relevée à gauche vers i & secouée \\
\hline $\mathrm{R}$ & en bas & $\mathrm{i}$ & $\mathrm{i}$ \\
\hline$S$ & & $\wedge$ & $x$ \\
\hline
\end{tabular}




\begin{tabular}{rccl}
\hline 13. & je dis les choses & telles qu'elles sont- & \\
\hline $\mathrm{P}$ & & (.) & \\
$\mathrm{T}$ & mvt trans. à gauche & repos & \\
$\mathrm{R}$ & $\mathrm{i}$ & $\mathrm{i}$ & fixe sur i \\
$\mathrm{B}$ & & & lèvres pincées
\end{tabular}

\begin{tabular}{rccc}
\hline 14. & oùtout le monde & n'a pas les mêmesscru- & pules, \\
\hline $\mathrm{P}$ & & & (.) \\
$\mathrm{T}$ & mvt.trans. rapide & fortementabaissée & repos \\
$\mathrm{R}$ & $\mathrm{i}$ & $\mathrm{i}$ & \\
$\mathrm{B}$ & & &
\end{tabular}

\begin{tabular}{|c|c|c|c|}
\hline 15. & où tous les coups & peuvent être donnés & \\
\hline$P$ & & 1 & (.) \\
\hline $\mathrm{T}$ & abaissée & et plus faiblement deux fois & \\
\hline $\mathrm{R}$ & $\mathrm{i}$ & $\mathrm{i}$ & \\
\hline $\mathrm{S}$ & $\wedge$ & $\wedge$ & \\
\hline B & sourire & & ;) \\
\hline
\end{tabular}

\begin{tabular}{ccc}
\hline 16. & et où, pourabattre quelqu'un, & \\
\hline $\mathrm{P}$ & \multicolumn{1}{c}{$/$} & $()$. \\
$\mathrm{T}$ & penchée à droite & \\
$\mathrm{R}$ & $\mathrm{i}$ \\
$\mathrm{S}$ & $\wedge \wedge$ & \\
$\mathrm{B}$ & & \\
& &
\end{tabular}

\begin{tabular}{|c|c|c|}
\hline 17. & on utilise & tous les procédés. \\
\hline$P$ & & (.) \\
\hline $\mathrm{T}$ & recentrée & abaissée \\
\hline$B$ & & (:) \\
\hline
\end{tabular}

\begin{tabular}{ccc}
\hline 18. & Je reste serEIN & etje reste calme. \\
\hline $\mathrm{P}$ & / & I \\
$\mathrm{T}$ & centrée abaissée & relevée, tournée vers i \\
$\mathrm{B}$ & en bas & $\mathrm{i}$
\end{tabular}

\begin{tabular}{cccc}
\hline 19. & Je FAIS CET engagement & qui est le mien & depuis bien longtemps. \\
\hline $\mathrm{P}$ & $\mathrm{J}$ & & $/$ \\
$\mathrm{T}$ & mvt d'appui vers le bas & repos & penchement à gauche \\
$\mathrm{B}$ & $\mathrm{i}$ & $\mathrm{i}$ & $\mathrm{i}$
\end{tabular}




\begin{tabular}{|c|c|c|c|}
\hline 20. & RIEN & ne me détournera de la route que & j'ai choisie. \\
\hline$P$ & & / & (.) \\
\hline $\mathrm{T}$ & mvt transv & immobile & immobile \\
\hline $\mathrm{R}$ & i & $\mathrm{i}$ & i \\
\hline$S$ & & & $\wedge \wedge$ \\
\hline
\end{tabular}

\section{Analyse}

Nous allons voir que les mouvements de la tête non seulement découpent l'énoncé, désignent des éléments présents ou absents, produisent des postures physiques qui expriment des attitudes, mais encore produisent des mouvements qui représentent concrètement des notions abstraites. Bref, la mobilité de la tête permet d'assurer de multiples fonctions.

\section{Permanence de la fonction démarcative kinésique}

La fonction démarcative s'exprime par la segmentation hiérarchisée, corporelle et sémantique du propos.

\section{Unités temporelles}

Dans l'extrait 1, le mouvement ascendant-descendant de la tête qui rythme chacune des trois premières unités temporelles «colle» à la courbe intonative, ascendante-descendante.

Par contre, dans l'extrait 3, le mouvement général qui rythme les annonces des deux idées plus importantes qu'il explicitera ensuite - dans ce monde d'agressions (segment 12...), il « reste serein » (segment 18...) -, consiste, pour Nicolas Sarkozy, à les aborder avec la tête et le regard baissés, comme pour mieux se concentrer (etmalheureusement... ; je reste serein...), avant de relever la tête et de diriger le regard vers l'interlocuteur comme pour lui adresser, lui inculquer visuellement ce qu'il a à lui dire (tête et regard baissés) : et malheureusement (relevés vers l'interlocuteur), nous vivons dans un monde...; (baissés) je reste serein (relevés) et je reste calme...

\section{Sous-unités}

À quatre reprises durant un passage lourd de sens, Nicolas Sarkozy segmente les groupes rythmico-sémantiques par ce que l'on pourrait appeler un sourire devant l'adversité, un sourire «fermé » caractérisé par un relèvement marqué des commissures, sans découvrir les dents ni plisser les yeux qui ne sourient guère. Voir à ce propos le signe :) qui termine chacun des segments 14 à 17 . 
La mimique buccale expressive ponctue rapidement chaque segment comme si l'idée d'attaque parallèlement énoncée, et même de plaisir à attaquer exprimée par cette mimique, était maintenue constante durant tout ce passage et ne pouvait se manifester qu'au moment d'une brève pause entre les groupes rythmiques.

On remarque de manière générale que la tête et les muscles du visage de Nicolas Sarkozy sont toujours mobiles, sauf à deux moments où il parle de sa femme Cécilia. Il s’immobilise alors, regard fixe devant lui, pour nous signifier ce qui compte le plus pour lui. Le figement de la posture se produit aux moments clés: quand il avoue «devoir énormément »à sa femme (segment 6) et, après une incise relative à son amour envers elle, quand il dit penser que cet amour est partagé (segment 8). Il s’immobilisera à nouveau à la fin, pour mettre en exergue et figurer, par l'immobilité de la tête, qu'il ne se détournera pas de la route qu'il s'est fixée (20). Par le contraste postural et la sobriété de la posture qu'il adopte comme si, se parlant à lui-même, il se confessait publiquement, Nicolas Sarkozy attire l'attention sur le contenu sémantique de l'énoncé qui, par là même, devient important.

Bien que mon propos soit centré sur la fonction démarcative, on aura remarqué que le mouvement de tête, allié à la direction du regard vers le bas puis vers l'interlocuteur, assure simultanément une autre fonction, expressive en mimant la concentration, puis phatique en s'adressant à l'interlocuteur qui va bénéficier du produit de la réflexion (segments 12,18 ). Comme il assure simultanément deux fonctions, il est ici multifonctionnel.

\section{Autres fonctions, par mouvement de tête}

Je prends désormais le parti de rendre compte des mouvements de la tête en les regroupant, non par fonction exercée mais par type de mouvement effectué pour mieux faire apparaître, à partir de sa modulation physique, ses nombreuses possibilités symboliques.

Un mouvement de la tête peut aussi bien désigner un point situé dans son prolongement que reproduire une posture ou figurer concrètement une notion. Il change alors de fonction. Voici divers types de mouvements de tête dont on pourra constater pour chacun la multifonctionnalité, d'une part, et la polysémie (plusieurs significations) d'autre part. Leur ordre de présentation s'appuie sur une classification sous-jacente des mouvements de tête en ligne courbe:

- en plan sagittal, vers le haut (Relèvement) ou vers le bas (Abaissement),

- en plan frontal, en penchant la tête vers l'épaule, droite ou gauche (Penchement),

- en plan horizontal, sur l'axe transversal par un mouvement sur le côté, aussi bien pour regarder, aller vers... (Rotation pour se tourner vers) que 
pour se détourner de... (Rotation pour se détourner de), c'est-à-dire pour respectivement désigner et situer quelque chose ou bien refuser ce qui est proposé. Le mouvement de rotation se répète de part et d'autre (Secouement latéral de la tête) ou commence d'un côté pour mieux aller de l'autre (Mouvement transversal de la tête).

\section{Relèvement}

Dans l'extrait 1, le relèvement de la tête est porteur d'une signification, différente dans chaque phrase et toujours analogiquement motivée. Ce relèvement est à interpréter comme la représentation d'un cumul et un signe d'exclamation augmentative s'il est accompagné d'une mélodie ascendante, et surtout confirmé par un haussement de sourcils portant sur le mot clé: des millions (segment 1).

Conséquence d'un relèvement du tronc dont la tête est le prolongement, le mouvement de tête vers le haut sera, par contre, à interpréter comme la figuration euphémique du cabrage du corps entier qui se redresse pour mieux affronter les difficultés: ces difficultés, nous sommes en train de les... (3).

Dans la troisième phrase, les deux relèvements de tête successifs exprimeraient deux interrogations: yeux fermés et sourcils levés, d'abord une question à soi-même sur le fait de devoir parler, suivie d'une question à l'interlocuteur regardé et interpellé du menton sur le bien fondé d'en dire plus: (montée) est-ce que je dois (descente) en dire (montée) plus? Le geste coverbal participe de l'interrogation par une deixis d'interpellation (4).

On voit là que l'interprétation du mouvement se déduit d'une confrontation entre les divers éléments du contexte, qu'il soit vocal, kinésique ou textuel.

\section{Abaissement}

La force et l'amplitude d'un mouvement d'abaissement sont des éléments physiques pertinents qui en modifient la signification. La répétition du mouvement elle-même interfère avec les données précédentes. Voyons les divers cas rencontrés:

Force du mouvement. - Un seul mouvement marqué vers le bas, et donc perçu comme un appui, exprime l'insistance sur la chose simultanément énoncée. C'est ainsi que Nicolas Sarkozy dénonce la médisance ou la calomnie en appuyant physiquement sur le fait de dire: (je ne pense pas que cela mérite) qu'on dise (des choses) (9). De la même façon, dans le segment 14 - tout le monde n'a pas les mêmes scrupules -, l'apogée du mouvement coïncide avec la prononciation du mot mêmes. Cette insistance kinésique sur la non-équivalence semble sous-entendre : $n$ 'a pas les mêmes scrupules 'que moi'. Pareillement, à la fin de son intervention, il va, avec la tête, insister sur 
son engagement - je fais cet engagement (19) -, auquel il donne, par là même, un caractère solennel. Deux fois sur trois, l'accentuation kinésique se double d'un accent vocal: QU'ON DISE des (9); je FAIS CET engagement (19).

Force et répétition. - Si le mouvement vers le bas exprime l'insistance, sa réitération est susceptible de produire une scansion insistante : (on dit des choses) qui lui manquent beaucoup de respect (11). Ce commentaire muet d'insistance sur le manque de respect est confirmé par une pression des lèvres provoquant l'étirement visible des commissures. Nicolas Sarkozy produit cette mimique lors de deux moments d'appréciation : appréciation de ce qu'il doit à sa femme (6), appréciation du manque de respect qu'elle a subi (11). Autrement dit, Nicolas Sarkozy confirme ses appréciations orales par des mimiques buccales.

Répétition d'un mouvement sans force. - Sans force, le mouvement vers le bas ne peut exprimer l'appui. Qu'est-ce donc? Signe partagé d'acceptation en Occident et signifiant Oui hors contexte, l'inclination de la tête vers le bas serait un dérivé euphémique de la posture de soumission (Morris, 1977) ou encore une posture de repli sur soi, signalant un moment de déconnectionréflexion. Indiquant l'acquiescement, la confirmation, ce peut être dans le cas présent un signe d'autoconfirmation. De la tête, le locuteur confirme ce qu'il est en train d'énoncer. Le geste commente alors silencieusement ce qui est dit: où tous les coups 'eh oui' (15). Comme cette phrase n'est pas finie, la répétition du mouvement de confirmation va permettre aussi d'en scander la fin: (abaissement) où tous les coups (répété deux fois) peuvent être donnés (15). Poursuivant son idée, Nicolas Sarkozy reconfirme son point de vue d'un nouvel abaissement de tête: et où, pour abattre quelqu'un (16), on utilise (à nouveau, tête abaissée) tous les procédés (17).

\section{Penchement}

Là encore, le mouvement est susceptible de désigner, de reproduire ou de figurer quelque chose (voir supra: «Quelques clés pour l'analyse gestuelle»). Dans la vie courante, on penche la tête sur le côté pour envisager un objet sous divers angles. Reproduire ce penchement et la maintenir un temps permet, par la figuration d'un angle de vision, d'évoquer un point de vue particulier, le point de vue personnel: je ne pense pas que... (9).

Dans un autre contexte, le penchement est susceptible de figurer la recherche visuelle du meilleur angle d'attaque: pour abattre quelqu'un (16). On notera que dans un combat, ici évoqué de façon métaphorique et euphémique dans la conversation, chercher à se protéger et à voir tout de même les mouvements de l'adversaire aboutit à la même posture, tête penchée, regard par en dessous.

Ces deux penchements de tête se sont faits à droite. La troisième s'effectue à gauche (18). S'agit-il encore de la représentation d'un angle de vue? 
La confrontation avec l'énoncé depuis bien longtemps (18) nous indique qu'il s'agit du passé. Or le passé est situé à gauche sur l'axe orienté du temps objectif: l'axe transversal rend compte de la suite des évènements constatés, assimilables à des objets juxtaposés et contemplés devant soi. Conformément au sens de l'écriture en Occident, cette suite logico-temporelle est symboliquement représentée de gauche à droite (Calbris, 1985, 2003, p. 183-186). Ce qui veut dire qu'ici, le penchement de la tête ne reproduit plus une posture mais désigne une zone. Il ne s'agit plus du résultat du mouvement, d'une mise en posture, mais du prolongement fictif du mouvement vers un endroit de l'espace-temps.

La cause de ces penchements est bien sémantique et non ergonomique. Leur direction ne dépend pas de la position de tête adoptée pour mieux s'adresser à l'interlocuteur. En effet, Nicolas Sarkozy effectue deux penchements dans la même direction, à droite ( 9 et 16), alors qu'il répond à Stéphane Lippert situé sur sa gauche (en 9), puis à Jean-Michel Blier, plutôt sur sa droite (en 16). C'est aussi en s'adressant au même journaliste qu'il effectue deux penchements opposés, à droite (16) et à gauche (18).

\section{Rotation pour se tournervers}

On se tournevers quelqu'un (représentant éventuellement quelqu'un d'autre), vers un endroit symbolique ou vers un objet abstrait symboliquement situé à cet endroit. La tête s'oriente vers un point que le prolongement fictif du mouvement désigne comme étant sur le côté ou juste derrière soi.

Comme tout Français, Nicolas Sarkozy fixe l'interlocuteur du regard pour qu'il retienne ce qu'il estime important et, dans ce but, tourne la tête vers lui : (la vérité) elle est très simple (1); (Cécilia) compte beaucoup pour moi (7); (on dit des choses) qui la font souffrir (11). Les mouvements délibérés et conjoints de la tête et du regard désignent le destinataire du propos, le journaliste ou le public-camera(man).

Nicolas Sarkozy tourne aussi la tête pour désigner un endroit dont la situation relative sur le côté de son corps, pris comme référence, est entachée d'une signification symbolique physiquement motivée. Désignant ce qui est physiquement à côté de lui, Nicolas Sarkozy signale la position symboliquement annexe de l'incise verbale : on dit des choses, la plupart du temps d'ailleurs... inexactes (10). Voici un autre exemple plus complexe d'énoncé donné en incise. Nicolas Sarkozy commence par un constat important - Cécilia, je lui dois énormément (6) - dont il donne la résultante affective - Cécilia compte beaucoup pour moi - mais en incise (7). La déclaration est publique (sourire au public regardé à travers l'œil de la caméra). Si Nicolas Sarkozy insiste sur cette reconnaissance - Cécilia (compte beaucoup pour moi) - par un mouvement d'appui de la tête vers le bas, il la considère néanmoins comme une infor- 
mation devant rester annexe de la part d'un politicien, puisque le mouvement d'appui se fait sur le côté et non devant soi. Insistant sur la reconnaissance (appui), il la désigne comme une incursion privée et donc annexe (à gauche).

Nous avons affaire à un geste complexe qui, en combinant deux mouvements, assure deux fonctions, une fonction expressive d'insistance et une fonction référentielle métacommunicative. De manière générale, l'incise est accompagnée d'un mouvement de la tête vers le côté - ici, le côté gauche -, mouvement qui aurait pu être effectué en plan frontal et être accompagné d'un déploiement parallèle de la main sur le côté (Calbris, 1983, p. 736-737). Elle est aussi parfois signalée par un changement de main maintenu tout le temps de son énonciation recto tono pour mieux isoler, par un décroché intonatif, le segment concerné (Calbris, 2003, p. 170). Elle est donc, dans tous les cas, située sur le côté. L'incise verbale semble bien, d'après l'indice gestuel cooccurrent et récurrent, mentalement conçue comme un à côté par rapport au projet du discours conçu comme une route sur laquelle on avance (Lakoff, Johnson, 1980; Sweetser, 1998). Le contenu sémantique de l'incise, cet objet abstrait annexe, est signalé comme tel et physiquement désigné sur le côté.

\section{Rotation pour se détourner de quelque chose}

La rotation de la tête et du visage permet d'éviter la source d'un désagrément gustatif (Morris, p. 50), olfactif (Darwin, 1981, p. 249) ou visuel. Réflexe d'évitement d'un désagrément, cette rotation devient un signe de refus.

Le corpus présente plusieurs mouvements complexes dont l'un combine un fort penchement à droite, allié à un détournement à gauche : je ne le pense... pas (5). Comme en (9), je ne pense pas que, le penchement en (5), durant la phrase je ne le pense... pas, exprime le point de vue personnel sur la chose évoquée. Par contre, le détournement simultanément effectué à gauche exprime, lui, la réponse à la proposition précédemment évoquée, est-ce que je dois en dire plus? Ce sera non : je ne le pense... pas. Ainsi, le penchement (qui figure un angle de vue sur la chose) exprime le point de vue tandis que le détournement de la tête (qui figure le réflexe d'évitement) exprime l'appréciation négative qui en résulte, et le refus de l'objet abstrait considéré. Ce geste complexe a deux significations (point de vue, refus) qui, déduites des composantes cognitives qui sous-tendent l'enchainement discursif, reposent sur deux composants physiques (penchement; rotation). Ces deux composants physiques supportent respectivement le lien analogique (angle de vue; réflexe d'évitement) établi entre la signification contextuelle et l'élément physique correspondant: à savoir le point de vue analogiquement signifié par un angle de vue produit par un penchement de tête, d'une part; le refus signifié par la reproduction du réflexe d'évitement qui consiste à détourner la tête de l'objet non voulu, d'autre part. 
La comparaison des images correspondant à je ne le pense... pas (5) et à je ne pense pas que (9) est éclairante. Le penchement commun représente physiquement le point de vue, mental, alors que le mouvement complémentaire d'évitement par rotation sur le côté exprime le point de vue négatif que l'énoncé corrobore : je ne le pense... pas.

\section{Secouement latéral de la tête}

Signe explicite de refus pour les Français, comme pour de nombreux autres groupes culturels, le secouement latéral de la tête est à interpréter physiquement comme un évitement répété de l'offre réitérée face au visage, successivement déplacé d'un côté, puis de l'autre (Calbris, 1990, p. 131-133).

Contrairement à ce que l'on pourrait supposer d'emblée, ce signe de refusnégation n'illustre pas la négation verbale «ne pas». Il suffit de se reporter aux segments d'énoncés contenant une négation verbale - je ne pense pas que (9); tout le monde n'a pas les mêmes scrupules (14) - pour constater qu'ils sont accompagnés de mouvements de tête autres que le secouement latéral. Ce qui veut dire que le signe de tête n'est pas redondant par rapport à l'énoncé. Il a une signification physique qui lui est propre.

Le secouement latéral de la tête apparait comme une expression physique de refus par rapport à un objet abstrait (Calbris, 2005). C'est bien le cas quand Nicolas Sarkozy affirme: comme des millions de familles, la mienne a connu des difficultés (2). Le geste qui sous-entend 'la mienne n'est pas différente' exprime le refus de l'idée reçue par les spectateurs d'une famille extraordinaire qui serait différente de la leur. Il est accompagné d'un petit sourire qui peut paraitre auto-ironique et amusé.

En (12), Nicolas Sarkozy explique: et malheureusement, nous vivons dans un monde... Monde difficile à vivre et donc à rejeter a priori. Le secouement de tête vient exprimer le refus du rejet sous-entendu, simultanément confirmé par je ne m'en plains pas (12). Compte tenu de l'enchainement des énoncés, le geste qui refuse un sous-entendu logique n'est pas, en soi, l'illustration de la négation verbale je ne m'en plains pas. Complément expressif, le ton plaintif de cet énoncé exprimerait combien cette acceptation est résignée.

\section{Mouvement transversal de la tête}

Allant d'un côté à l'autre, la tête exécute une rotation transversale rapide. Ce peut être une des variantes de la totalité des gestes qui, effectués avec la main et / ou la tête, présententen commun un mouvementtransversal.Celui-cibalaie l'horizon et, à travers le percept du 'partout', réfère à la notion de totalité (Calbris, 1990, p. 131-132) : malheureusement nous vivons dans un monde [...] où tout le monde n'a pas les mêmes scrupules (14). On retrouve la même expres- 
sion et la même idée lorsque Nicolas Sarkozy déclare que rien ne le détournera de la route choisie (20). Le mouvement qui balaie l'horizon permet de parcourir du regard l'ensemble des individus (14) ou tout élément susceptible de contrecarrer son projet (20).

On constate que les segments d'énoncé suivants, qui réfèrent verbalement à la totalité - où tous les coups (15); tous les procédés (17) -, ne sont pas accompagnés de ce mouvement transversal de la tête, mais du mouvement vers le bas dont la signification et la motivation ont été données ci-dessus. Ce n'est donc pas la notion énoncée de totalité qui détermine le geste qui la figure, mais le besoin de concrétiser la notion qui détermine le geste. Celui-ci vient, de façon expressive, compléter simultanément l'énoncé.

La totalité n'est pas la seule acception possible du mouvement transversal de la tête. Souvent synchrone du mouvement transversal de la main horizontale en pronation auquel il se superpose, il est polysémique et peut signifier, par le fait d'aller directement, en ligne droite, de tel endroit à tel endroit sans arrêt ni détour, le caractère direct de..., c'est-à-dire aussi bien la franchise de quelqu'un que le caractère inéluctable et certain de l'évènement. Le geste paraphrase de façon expressive l'énoncé: ('directement, sans ambages') je dis les choses telles qu'elles sont (13); ('directement, de façon suivie') on utilise tous les procédés (17).

Ce corpus donne un bel échantillon du transfert possible de la diversité de l'expression non verbale sur un simple segment corporel, la tête. On constate que la mobilité restreinte de la celle-ci n'empêche pas la réalisation des différentes fonctions communicatives et énonciatives assurées par le corps, car elle permet aussi bien de segmenter l'énoncé, de maintenir un contact avec le destinataire, d'exprimer une attitude que de désigner un référent ou même de le figurer par un «schéma imagier mental» (Turner, 2000) extrait de notre expérience corporelle.

On constate aussi qu'un mouvement de la tête ne correspond ni à une seule fonction (geste multifonctionnel) ni à une seule signification (geste polysémique). Un mouvement de tête peut aussi combiner deux composants physiques susceptibles de supporter chacun un lien analogique et, par là même, de figurer simultanément deux notions (geste polysigne biréférentiel; Calbris, 2003).

L'interprétation d'un mouvement de tête, comme de tout code analogique et polysémique, est éminemment contextuelle. Son interprétation dépend d'une convergence de données, de celles fournies par le contexte - verbal, vocal, kinésique - comme de celles obtenues plus tôt dans le discours. Le contexte interprétatif est à la fois synchronique et diachronique.

Une récapitulation très brève des résultats de l'analyse des mouvements de tête nous montre que le geste n'est pas une illustration redondante de la parole : 
par exemple, l'expression verbale de la totalité n'est pas nécessairement doublée de sa représentation figurative; ni l'emploi de la forme négative, accompagnée d'un refus de la tête. Et inversement, un refus de la tête n'est pas nécessairement prononcé. L'expression kinésique n'est donc pas superfétatoire.

Nous avons vu qu'elle assure plusieurs fonctions. Elle sert à la segmentation hiérarchisée du discours (fonction démarcative, supra). Constamment mobile, la tête va maintenir et diriger l'attention (fonction phatique) sur certains segments du discours $(6,8,20)$, simplement en s'immobilisant le temps de l'émission sonore du segment. La tête permet d'exprimer une attitude (fonction expressive), un point de vue négatif par exemple, verbalement confirmé par je ne le pense pas. Bel exemple d'expression non verbale superfétatoire, dira-t-on? II n'en est rien. Dans la pragmatique du discours, on voit l'expression physique du point de vue personnel atténuer, de par l'introduction visuelle de la subjectivité, la valeur négative de la réponse à la question posée: je ne le pense pas. En outre, la tête permet de situer des objets mentaux comme de figurer des notions abstraites, de façon stylisée dans l'espace (fonction référentielle). Par exemple, comme tout un chacun, Nicolas Sarkozy situe physiquement sur le côté un propos qu'il considère comme une incise verbale, ce qui signifie par analogie que l'objet mental en question est conçu et représenté comme un élément devant être annexe par rapport au projet central du discours (7 et 10).

Compte tenu de l'intrication des informations référentielles sonores et kinésiques à l'oral, voici un essai de traduction des informations kinésiques faisant partie de l'énoncé (Code verbal et kinésique) :

La vérité, elle est très simple. Comme des millions de familles, la mienne 'elle n'est pas différente' a connu des difficultés. Ces difficultés 'j’y fais face', nous sommes en train de les surmonter. Est-ce que je dois en dire plus? 'Selon mon angle de vue, c'est non' Je ne le pense pas.

Et malheureusement, nous vivons dans un monde - 'je ne le refuse pas' je ne m'en plains pas, je dis 'directement' les choses telles qu'elles sont - où tout le monde n'a pas les mêmes scrupules 'que moi', où tous les coups peuvent être donnés 'eh oui' et où, pour 'mieux' abattre quelqu'un, on utilise 'directement, de façon catégorique' tous les procédés, 'oui'.

Faisant partie intégrante du discours, les mouvements de tête étudiés sont en général des gestes non conscients et spontanés qui apparaissent au fur et à mesure des idées à exprimer oralement. La recherche de leur signification contextuelle et de leur motivation physique montre que le locuteur réagit physiquement à des objets mentaux conçus à l'image d'objets physiques. Ces signes coverbaux obéissent à un code analogique dont l'analyse révèle qu'il est basé sur une expérience perceptivo-motrice communément partagée.

On peut penser que des experts de la communication ont conseillé à Nicolas Sarkozy la sobriété et un « tête à tête » avec les Français concrétisé par 
le gros plan, et même imaginer un énoncé préparé, car l'énoncé transcrit est loin de l'improvisation orale. Cependant, pour ne pas réciter, le locuteur doit, comme tout acteur, se remettre en situation et s'efforcer de penser ce qu'il dit, s'accorder une marge de spontanéité pour rester authentique et ainsi faire passer le message, car la relation profonde entre le geste et la parole (McNeill) est essentielle à la production du sens et à sa compréhension.

En résumé, l'analyse du geste coverbal révèle que la signification propre du geste dérive de ses fonctions dans la vie courante ou reproduit de façon stylisée des éléments de notre expérience perceptivo-motrice qui sont à la base de notre conception de l'abstrait. Primaire et ambiguë, et propre à toute communication vivante, cette expression physique symbolique est précisée par le contexte. Ce dernier sélectionne une des potentialités symboliques du geste, révélées par l'analyse sémiologique de ses emplois contextuels.

\section{Références}

CALBRIS Geneviève, 1983, Contribution à une analyse sémiologique de la mimique faciale et gestuelle française dans ses rapports avec la communication verbale, 4 vol. (1. Expérimentation, 2. Taxinomie, 3. Synthèse, 4. Annexe illustrative), thèse d’État, 1478 p., 17 microfiches, http://catalogue.bibliotheque.msh-paris.fr

- 1985, «Espace-Temps. Expression gestuelle du temps», Semiotica, nº 55 (1/2), p. 43-73.

- 1987, "Geste et motivation », Semiotica, n 65 (1/ 2), p. 57-96.

- 1990, The Semiotics of French Gestures, Bloomington, Bloomington University Press.

- 2003, "Multireferentiality of coverbal gestures», Gestures: Their Meaning and Use, M. Rector, I. Poggi, N. Trigo éd., Porto, Fernando Pessoa University Press, p. 203207.

- 2003, L'expression gestuelle de la pensée d'un homme politique, Paris, CNRS (CNRS Communication), www.cnrseditions.fr

- 2005, "Lanégation. Son symbolisme physique », IIe ISGSConference, Lyon, 15-18 juin 2005, Interacting Bodies. Abstracts, p. 62.

Condon William S., 1984, "Une analyse de l'organisation comportementale», La communication non verbale, J. Cosnier, J. Brossard éd., p. 31-70 [1976, «An analysis of behavioral organisation », Sign Language Studies, n 13, p. 285-318].

DARWIN Charles, 1981, L'expression des émotions chez l'homme et les animaux, Bruxelles, Complexe [1872, The Expression of the Emotions in Man and Animals, Londres, John Murray].

KENDON Adam, 1972, "Some relationships between body motion and speech. An analysis of an example», Studies in Dyadic Communication, A. Siegman, B. Pope éd., Elmsford, New York, Pergamon Press, p. 177-210.

-2002 , "Some uses of the head shake», Gesture, $n^{\circ} 2$ (2), p. 147-182. 


\section{GeneVIÈVE CALbris}

LAKoff George, Johnson Mark, 1980, Metaphors We Live by, Chicago, IL, University of Chicago Press.

MCCLAVE Evelyn, 2005, "Cognitive universals: evidence from head movements in the context of speech », IIe ISGS Conference, Lyon, 15-18 juin 2005, Interacting Bodies. Abstracts, p. 128.

McNeILl David, 1992, Hand and Mind. What Gestures Reveal about Thought, Chicago, Chicago University Press.

MontAgner Henri, 1978, L'enfant et la communication, Paris, Stock (Laurence Pernoud). Morris Desmond, 1987, La clé des gestes, Paris, Grasset, 320 p. [1977, Manwatching. A Field Guide to Human Behaviour, New York, H. N. Abrams].

PARrill Fey, SWeEtSER Eve, 2004, «What we mean by meaning. Conceptual integration in gesture analysis and transcription », Gesture, ${ }^{\circ} 4$ (2), p. 197-219.

SWEETSER Eve, 1998, «Regular metaphoricity in gesture. Bodily-based models of speech interaction », Actes du 16 Congrès international des linguistes, CD-ROM, Elsevier.

TURner Mark, 2000, Conférences au Collège de France, $n^{\circ} 3$, «La perspicacité et la mémoire», http://markturner.org/cdf 